\title{
ON THE BRAUER GROUP OF ENRIQUES SURFACES
}

\author{
Arnaud Beauville
}

\begin{abstract}
Let $S$ be a complex Enriques surface (quotient of a K3 surface $X$ by a fixed-point-free involution). The Brauer group $\operatorname{Br}(S)$ has a unique nonzero element. We describe its pull-back in $\operatorname{Br}(X)$, and show that the surfaces $S$ for which it is trivial form a countable union of hypersurfaces in the moduli space of Enriques surfaces.
\end{abstract}

\section{Introduction}

Let $S$ be a complex Enriques surface, and $\pi: X \rightarrow S$ its 2-to-1 cover by a K3 surface. Poincaré duality provides an isomorphism $\mathrm{H}^{3}(S, \mathbb{Z}) \cong \mathrm{H}_{1}(S, \mathbb{Z})=\mathbb{Z} / 2$, so that there is a unique nontrivial element $b_{S}$ in the Brauer group $\operatorname{Br}(S)$. What is the pull-back of this element in $\operatorname{Br}(X)$ ? Is it nonzero? ${ }^{1}$

The answer to the first question is easy in terms of the canonical isomorphism $\operatorname{Br}(X) \stackrel{\sim}{\sim} \operatorname{Hom}\left(T_{X}, \mathbb{Q} / \mathbb{Z}\right)$ (see $\left.\S 2\right): \pi^{*} b_{S}$ corresponds to the linear form $\tau \mapsto\left(\beta \cdot \pi_{*} \tau\right.$ ), where $\beta$ is any element of $\mathrm{H}^{2}(S, \mathbb{Z} / 2)$ which does not come from $\mathrm{H}^{2}(S, \mathbb{Z})$. The second question turns out to be more subtle: the answer depends on the surface. We will characterize the surfaces $S$ for which $\pi^{*} b_{S}=0$ (Corollary 5.7), and show that they form a countable union of hypersurfaces in the moduli space of Enriques surfaces (Corollary 6.5).

Part of our results hold over any algebraically closed field, and also in a more general set-up (see Proposition 4.1 below); for the last part, however, we need in a crucial way Horikawa's description of the moduli space by transcendental methods.

\section{The Brauer group of a surface}

Let $S$ be a smooth projective variety over a field; we define the Brauer group $\operatorname{Br}(S)$ as the étale cohomology group $\mathrm{H}_{\text {êt }}^{2}\left(S, \mathbb{G}_{m}\right)$. For surfaces this definition coincides with that of Grothendieck [G] by [G], II, Cor. 2.2; this holds in fact in any dimension by a result of Gabber, which we will not need here (see $[\mathrm{dJ}]$ ).

In this section we assume that $S$ is a complex surface; we recall the description of $\operatorname{Br}(S)$ in that case - this is classical but not so easy to find in the literature. The Kummer exact sequence

$$
0 \rightarrow \mathbb{Z} / n \longrightarrow \mathbb{G}_{m} \longrightarrow \mathbb{G}_{m} \rightarrow 0
$$

gives rise to an exact sequence

$$
0 \rightarrow \operatorname{Pic}(S) \otimes \mathbb{Z} / n \longrightarrow \mathrm{H}^{2}(S, \mathbb{Z} / n) \stackrel{p}{\longrightarrow} \operatorname{Br}(S)[n] \rightarrow 0
$$

(we denote by $M[n]$ the kernel of the multiplication by $n$ in a $\mathbb{Z}$-module $M$ ).

\footnotetext{
Received by the editors May 19, 2009.

${ }^{1}$ The question is mentioned in [H-S], where the authors construct an Enriques surface $S$ over $\mathbb{Q}$ for which $\pi^{*} b_{S} \neq 0$ (see Cor. 2.8).
} 
On the other hand, the cohomology exact sequence associated to $0 \rightarrow \mathbb{Z} \rightarrow \mathbb{Z} \rightarrow \mathbb{Z} / n \rightarrow 0$ gives:

$$
0 \rightarrow \mathrm{H}^{2}(S, \mathbb{Z}) \otimes \mathbb{Z} / n \longrightarrow \mathrm{H}^{2}(S, \mathbb{Z} / n) \longrightarrow \mathrm{H}^{3}(S, \mathbb{Z})[n] \rightarrow 0
$$

Comparing (2.a) and (2.b) we get an exact sequence

$$
0 \rightarrow \operatorname{Pic}(S) \otimes \mathbb{Z} / n \longrightarrow \mathrm{H}^{2}(S, \mathbb{Z}) \otimes \mathbb{Z} / n \longrightarrow \operatorname{Br}(S)[n] \longrightarrow \mathrm{H}^{3}(S, \mathbb{Z})[n] \rightarrow 0 \text {. }
$$

Let $\mathrm{H}^{2}(S, \mathbb{Z})_{\mathrm{tf}}$ be the quotient of $\mathrm{H}^{2}(S, \mathbb{Z})$ by its torsion subgroup; the cup-product induces a perfect pairing on $\mathrm{H}^{2}(S, \mathbb{Z})_{\mathrm{tf}}$. We denote by $T_{S} \subset \mathrm{H}^{2}(S, \mathbb{Z})_{\mathrm{tf}}$ the transcendental lattice, that is, the orthogonal of the image of $\operatorname{Pic}(S)$. We have an exact sequence

$$
\operatorname{Pic}(S) \stackrel{c_{1}}{\longrightarrow} \mathrm{H}^{2}(S, \mathbb{Z}) \stackrel{u}{\longrightarrow} T_{S}^{*} \rightarrow 0
$$

where $u$ associates to $\alpha \in \mathrm{H}^{2}(S, \mathbb{Z})$ the cup-product with $\alpha$. Taking tensor product with $\mathbb{Z} / n$ and comparing with (2.c), we get an exact sequence

$$
0 \rightarrow \operatorname{Hom}\left(T_{S}, \mathbb{Z} / n\right) \longrightarrow \operatorname{Br}(S)[n] \longrightarrow \mathrm{H}^{3}(S, \mathbb{Z})[n] \rightarrow 0 ;
$$

or, passing to the direct limit over $n$,

$$
0 \rightarrow \operatorname{Hom}\left(T_{S}, \mathbb{Q} / \mathbb{Z}\right) \longrightarrow \operatorname{Br}(S) \longrightarrow \operatorname{Tors~}^{3}(S, \mathbb{Z}) \rightarrow 0
$$

\section{Algebraic topology of Enriques surfaces}

3.1. Let $S$ be an Enriques surface (over $\mathbb{C}$ ). We first recall some elementary facts on the topology of $S$. A general reference is [BHPV], ch. VIII.

The torsion subgroup of $\mathrm{H}^{2}(S, \mathbb{Z})$ is isomorphic to $\mathbb{Z} / 2$; its nonzero element is the canonical class $K_{S}$. Let $k_{S}$ denote the image of $K_{S}$ in $\mathrm{H}^{2}(S, \mathbb{Z} / 2)$. The universal coefficient theorem together with Poincaré duality gives an exact sequence

$$
0 \rightarrow \mathbb{Z} / 2 \stackrel{k_{S}}{\longrightarrow} \mathrm{H}^{2}(S, \mathbb{Z} / 2) \stackrel{v_{S}}{\longrightarrow} \operatorname{Hom}\left(\mathrm{H}^{2}(S, \mathbb{Z}), \mathbb{Z} / 2\right) \rightarrow 0
$$

where $v_{S}$ is deduced from the cup-product.

3.2. The linear form $\alpha \mapsto\left(k_{S} \cdot \alpha\right)$ on $\mathrm{H}^{2}(S, \mathbb{Z} / 2)$ vanishes on the image of $\mathrm{H}^{2}(S, \mathbb{Z})$, hence coincides with the map $\mathrm{H}^{2}(S, \mathbb{Z} / 2) \rightarrow \mathrm{H}^{3}(S, \mathbb{Z})=\mathbb{Z} / 2$ from the exact sequence $(2 . b)$. Note that $k_{S}$ is the second Stiefel-Whitney class $w_{2}(S)$; in particular, we have $\left(k_{S} \cdot \alpha\right)=\alpha^{2}$ for all $\alpha \in \mathrm{H}^{2}(S, \mathbb{Z} / 2)$ (Wu formula, see [M-S]).

3.3. The map $c_{1}: \operatorname{Pic}(S) \rightarrow \mathrm{H}^{2}(S, \mathbb{Z})$ is an isomorphism, hence (2.e) provides an isomorphism $\operatorname{Br}(S) \stackrel{\sim}{\longrightarrow} \operatorname{Tors~}^{3}(S, \mathbb{Z}) \cong \mathbb{Z} / 2$. We will denote by $b_{S}$ the nonzero element of $\operatorname{Br}(S)$.

Let $\pi: X \rightarrow S$ be the 2-to-1 cover of $S$ by a K3 surface. The aim of this note is to study the pull-back $\pi^{*} b_{S}$ in $\operatorname{Br}(X)$.

Proposition 3.4. The class $\pi^{*} b_{S}$ is represented, through the isomorphism $\operatorname{Br}(X) \stackrel{\sim}{\longrightarrow} \operatorname{Hom}\left(T_{X}, \mathbb{Q} / \mathbb{Z}\right)$, by the linear form $\tau \mapsto\left(\beta \cdot \pi_{*} \bar{\tau}\right)$, where $\bar{\tau}$ is the image of $\tau$ in $\mathrm{H}^{2}(X, \mathbb{Z} / 2)$ and $\beta$ any element of $\mathrm{H}^{2}(S, \mathbb{Z} / 2)$ which does not come from $\mathrm{H}^{2}(S, \mathbb{Z})$. 
Proof. Let $\beta$ be an element of $\mathrm{H}^{2}(S, \mathbb{Z} / 2)$ which does not come from $\mathrm{H}^{2}(S, \mathbb{Z})$, so that $p(\beta)=b_{S}(2 . a)$. The pull-back $\pi^{*} b_{S} \in \operatorname{Br}(X)$ is represented by $\pi^{*} \beta \in \mathrm{H}^{2}(X, \mathbb{Z} / 2)$ $\cong \mathrm{H}^{2}(X, \mathbb{Z}) \otimes \mathbb{Z} / 2$; its image in $\operatorname{Hom}\left(T_{X}, \mathbb{Z} / 2\right)$ is the linear form $\tau \mapsto\left(\pi^{*} \beta \cdot \bar{\tau}\right)$. Since $\left(\pi^{*} \beta \cdot \bar{\tau}\right)=\left(\beta \cdot \pi_{*} \bar{\tau}\right)$, the Proposition follows.

Part $(i)$ of the following Proposition shows that the class $\pi^{*} \beta \in \mathrm{H}^{2}(X, \mathbb{Z} / 2)$ which appears above is nonzero. This does not say that $\pi^{*} b_{S}$ is nonzero, as $\pi^{*} \beta$ could come from a class in $\operatorname{Pic}(X)-$ see $\S 6$.

Proposition 3.5. (i) The kernel of $\pi^{*}: \mathrm{H}^{2}(S, \mathbb{Z} / 2) \rightarrow \mathrm{H}^{2}(X, \mathbb{Z} / 2)$ is $\left\{0, k_{S}\right\}$.

(ii) The Gysin map $\pi_{*}: \mathrm{H}^{2}(X, \mathbb{Z}) \rightarrow \mathrm{H}^{2}(S, \mathbb{Z})$ is surjective.

Proof. To prove $(i)$ we use the Hochschild-Serre spectral sequence :

$$
E_{2}^{p, q}=\mathrm{H}^{p}\left(\mathbb{Z} / 2, \mathrm{H}^{q}(X, \mathbb{Z} / 2)\right) \Rightarrow \mathrm{H}^{p+q}(S, \mathbb{Z} / 2) .
$$

We have $E_{2}^{1,1}=0$, and $E_{\infty}^{2,0}=E_{2}^{2,0}=\mathrm{H}^{2}(\mathbb{Z} / 2, \mathbb{Z} / 2)=\mathbb{Z} / 2$. Thus the kernel of $\pi^{*}$ : $\mathrm{H}^{2}(S, \mathbb{Z} / 2) \rightarrow \mathrm{H}^{2}(X, \mathbb{Z} / 2)$ is isomorphic to $\mathbb{Z} / 2$. Since it contains $k_{S}$, it is equal to $\left\{0, k_{S}\right\}$.

Let us prove $(i i)$. Because of the formula $\pi_{*} \pi^{*} \alpha=2 \alpha$, the cokernel of $\pi_{*}$ : $\mathrm{H}^{2}(X, \mathbb{Z}) \rightarrow \mathrm{H}^{2}(S, \mathbb{Z})$ is a $(\mathbb{Z} / 2)$-vector space; therefore it suffices to prove that the transpose map

$$
{ }^{t} \pi_{*}: \operatorname{Hom}\left(\mathrm{H}^{2}(S, \mathbb{Z}), \mathbb{Z} / 2\right) \longrightarrow \operatorname{Hom}\left(\mathrm{H}^{2}(X, \mathbb{Z}), \mathbb{Z} / 2\right)
$$

is injective. This is implied by the commutative diagram

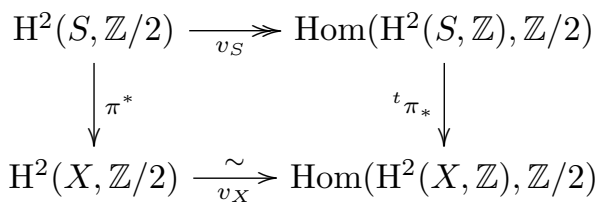

plus the fact that $\operatorname{Ker} \pi^{*}=\operatorname{Ker} v_{S}=\left\{0, k_{S}\right\}$ (by $(i)$ and (3.a)).

\section{Brauer groups and cyclic coverings}

Proposition 4.1. Let $\pi: X \rightarrow S$ be an étale, cyclic covering of smooth projective varieties over an algebraically closed field $k$. Let $\sigma$ be a generator of the Galois group $G$ of $\pi$, and let $\mathrm{Nm}: \operatorname{Pic}(X) \rightarrow \operatorname{Pic}(S)$ be the norm homomorphism. The kernel of $\pi^{*}: \operatorname{Br}(S) \rightarrow \operatorname{Br}(X)$ is canonically isomorphic to Ker Nm/(1- $\left.\sigma^{*}\right)(\operatorname{Pic}(X))$.

Proof. We consider the Hochschild-Serre spectral sequence

$$
E_{2}^{p, q}=\mathrm{H}^{p}\left(G, \mathrm{H}^{q}\left(X, \mathbb{G}_{m}\right)\right) \Rightarrow \mathrm{H}^{p+q}\left(S, \mathbb{G}_{m}\right) .
$$

Since $E_{2}^{2,0}=\mathrm{H}^{2}\left(G, k^{*}\right)=0$, the kernel of $\pi^{*}: \operatorname{Br}(S) \rightarrow \operatorname{Br}(X)$ is identified with $E_{\infty}^{1,1}=\operatorname{Ker}\left(d_{2}: E_{2}^{1,1} \rightarrow E_{2}^{3,0}\right)$. We have $E_{2}^{3,0}=\mathrm{H}^{3}\left(G, k^{*}\right)$; by periodicity of the cohomology of $G$, this group is canonically isomorphic to $\mathrm{H}^{1}\left(G, k^{*}\right)=\operatorname{Hom}\left(G, k^{*}\right)$, the character group of $G$, which we denote by $\widehat{G}$. So we view $d_{2}$ as a map from $\mathrm{H}^{1}(G, \operatorname{Pic}(X))$ to $\widehat{G}$. 
Let $\mathbf{S}$ be the endomorphism $L \mapsto \bigotimes_{g \in G} g^{*} L$ of $\operatorname{Pic}(X)$; recall that $\mathrm{H}^{1}(G, \operatorname{Pic}(X))$ is isomorphic to $\operatorname{Ker} \mathbf{S} / \operatorname{Im}\left(1-\sigma^{*}\right)$. We have $\pi^{*} \operatorname{Nm}(L)=\mathbf{S}(L)$ for $L \in \operatorname{Pic}(X)$, hence $\mathrm{Nm}$ maps $\operatorname{Ker} \mathrm{S}$ into $\operatorname{Ker} \pi^{*} \subset \operatorname{Pic}(S)$. Now recall that $\operatorname{Ker} \pi^{*}$ is canonically isomorphic to $\widehat{G}$ : to $\chi \in \widehat{G}$ corresponds the subsheaf $L_{\chi}$ of $\pi_{*} \mathcal{O}_{X}$ where $G$ acts through the character $\chi$. Since $\mathrm{Nm} \circ\left(1-\sigma^{*}\right)=0$, the norm induces a homomorphism $\mathrm{H}^{1}(G, \operatorname{Pic}(X)) \rightarrow \operatorname{Ker} \pi^{*} \cong \widehat{G}$. The Proposition will follow from:

Lemma 4.2. The map $d_{2}: \mathrm{H}^{1}(G, \operatorname{Pic}(X)) \rightarrow \widehat{G}$ coincides with the homomorphism induced by the norm.

Proof. We apply the formalism of [S], Proposition 1.1, where a very close situation is considered. This Proposition, together with property (1) which follows it, tells us that $d_{2}$ is given by cup-product with the extension class in $\operatorname{Ext}_{G}^{2}\left(\operatorname{Pic}(X), k^{*}\right)$ of the exact sequence of $G$-modules

$$
1 \rightarrow k^{*} \longrightarrow R_{X}^{*} \rightarrow \operatorname{Div}(X) \rightarrow \operatorname{Pic}(X) \rightarrow 0,
$$

where $R_{X}$ is the field of rational functions on $X$. This means that $d_{2}$ is the composition

$$
\mathrm{H}^{1}(G, \operatorname{Pic}(X)) \stackrel{\partial}{\longrightarrow} \mathrm{H}^{2}\left(G, R_{X}^{*} / k^{*}\right) \stackrel{\partial^{\prime}}{\longrightarrow} \mathrm{H}^{3}\left(G, k^{*}\right)
$$

where $\partial$ and $\partial^{\prime}$ are the coboundary maps associated to the short exact sequences

and

$$
\begin{gathered}
0 \rightarrow R_{X}^{*} / k^{*} \rightarrow \operatorname{Div}(X) \rightarrow \operatorname{Pic}(X) \rightarrow 0 \\
0 \rightarrow k^{*} \rightarrow R_{X}^{*} \rightarrow R_{X}^{*} / k^{*} \rightarrow 0 .
\end{gathered}
$$

Let $\lambda \in \mathrm{H}^{1}(G, \operatorname{Pic}(X))$, represented by $L \in \operatorname{Pic}(X)$ with $\perp_{g \in G} g^{*} L \cong \mathcal{O}_{X}$. Let $D \in \operatorname{Div}(X)$ such that $L=\mathcal{O}_{X}(D)$. Then $\sum_{g} g^{*} D$ is the divisor of a rational function $\psi \in R_{X}^{*}$, whose class in $R_{X}^{*} / k^{*}$ is well-defined. This class is invariant under $G$, and defines the element $\partial(\lambda) \in \mathrm{H}^{2}\left(G, R_{X}^{*} / k^{*}\right)$. Since $\operatorname{div} \psi$ is invariant under $G$, there exists a character $\chi \in \widehat{G}$ such that $g^{*} \psi=\chi(g) \psi$ for each $g \in G$. Then $d_{2}^{1,1}(\lambda)=\chi$ viewed as an element of $\mathrm{H}^{3}\left(G, k^{*}\right)=\widehat{G}$.

It remains to prove that $\mathcal{O}_{S}\left(\pi_{*} D\right)=L_{\chi}$. Since $\operatorname{div}(\psi)=\pi^{*} \pi_{*} D$, multiplication by $\psi$ induces a global isomorphism $u: \pi^{*} \mathcal{O}_{S}\left(\pi_{*} D\right) \stackrel{\sim}{\longrightarrow} \mathcal{O}_{X}$. Let $\varphi \in R_{X}$ be a generator of $\mathcal{O}_{X}(D)$ on an open $G$-invariant subset $U$ of $X$. Then $\operatorname{Nm}(\varphi)$ is a generator of $\mathcal{O}_{S}\left(\pi_{*} D\right)$ on $\pi(U)$, and $\pi^{*} \operatorname{Nm}(\varphi)$ is a generator of $\pi^{*} \mathcal{O}_{S}\left(\pi_{*} D\right)$ on $U$; the function $h:=\psi \pi^{*} \operatorname{Nm}(\varphi)$ on $U$ satisfies $g^{*} h=\chi(g) h$ for all $g \in G$. This proves that the homomorphism $u^{b}: \mathcal{O}_{S}\left(\pi_{*} D\right) \rightarrow \pi_{*} \mathcal{O}_{X}$ deduced from $u$ maps $\mathcal{O}_{S}\left(\pi_{*} D\right)$ onto the subsheaf $L_{\chi}$ of $\pi_{*} \mathcal{O}_{X}$, hence our assertion.

We will need a complement of the Proposition in the complex case:

Corollary 4.3. Assume $k=\mathbb{C}$, and $\mathrm{H}^{1}\left(X, \mathcal{O}_{X}\right)=\mathrm{H}^{2}\left(S, \mathcal{O}_{S}\right)=0$. The following conditions are equivalent:

(i) The map $\pi^{*}: \operatorname{Br}(S) \rightarrow \operatorname{Br}(X)$ is not injective;

(ii) there exists $L \in \operatorname{Pic}(X)$ whose class $\lambda=c_{1}(L)$ in $\mathrm{H}^{2}(X, \mathbb{Z})$ satisfies $\pi_{*} \lambda=0$ and $\lambda \notin\left(1-\sigma^{*}\right)\left(\mathrm{H}^{2}(X, \mathbb{Z})\right)$.

Observe that the hypotheses of the Corollary are satisfied when $S$ is a complex Enriques surfaces and $\pi: X \rightarrow S$ its universal cover. 
Proof. By Proposition $4.1(i)$ is equivalent to the existence of a line bundle $L$ on $X$ with $\operatorname{Nm}(L)=\mathcal{O}_{S}$ and $[L] \neq 0$ in $\mathrm{H}^{1}(G, \operatorname{Pic}(X))$, while $(i i)$ means that there exists such $L$ with $\left[c_{1}(L)\right] \neq 0$ in $\mathrm{H}^{1}\left(G, \mathrm{H}^{2}(X, \mathbb{Z})\right)$. Therefore it suffices to prove that the map

$$
\mathrm{H}^{1}\left(c_{1}\right): \mathrm{H}^{1}(G, \operatorname{Pic}(X)) \rightarrow \mathrm{H}^{1}\left(G, \mathrm{H}^{2}(X, \mathbb{Z})\right)
$$

is injective.

Since $\mathrm{H}^{1}\left(X, \mathcal{O}_{X}\right)=0$ we have an exact sequence

$$
0 \rightarrow \operatorname{Pic}(X) \stackrel{c_{1}}{\longrightarrow} \mathrm{H}^{2}(X, \mathbb{Z}) \longrightarrow Q \rightarrow 0 \quad \text { with } Q \subset \mathrm{H}^{2}\left(X, \mathcal{O}_{X}\right) .
$$

Since $\mathrm{H}^{2}\left(S, \mathcal{O}_{S}\right)=0$, there is no nonzero invariant vector in $\mathrm{H}^{2}\left(X, \mathcal{O}_{X}\right)$, hence in $Q$. Then the associated long exact sequence implies that $\mathrm{H}^{1}\left(c_{1}\right)$ is injective.

\section{More algebraic topology}

5.1. As in $\S 3$, we denote by $S$ a complex Enriques surface, by $\pi: X \rightarrow S$ its universal cover and by $\sigma$ the corresponding involution of $X$. We will need some more precise results on the topology of the surfaces $X$ and $S$. We refer again to [BHPV], ch. VIII.

Let $E$ be the lattice $\left(-E_{8}\right) \oplus H$, where $H$ is the rank 2 hyperbolic lattice. Let $\mathrm{H}^{2}(S, \mathbb{Z})_{\mathrm{tf}}$ be the quotient of $\mathrm{H}^{2}(S, \mathbb{Z})$ by its torsion subgroup $\left\{0, K_{S}\right\}$. We have isomorphisms

$$
\mathrm{H}^{2}(S, \mathbb{Z})_{\mathrm{tf}} \cong E \quad \mathrm{H}^{2}(X, \mathbb{Z}) \cong E \oplus E \oplus H
$$

such that $\pi^{*}: \mathrm{H}^{2}(S, \mathbb{Z})_{\mathrm{tf}} \rightarrow \mathrm{H}^{2}(X, \mathbb{Z})$ is identified with the diagonal embedding $\delta$ : $E \hookrightarrow E \oplus E$, and $\sigma^{*}$ is identified with the involution

$$
\rho:\left(\alpha, \alpha^{\prime}, \beta\right) \mapsto\left(\alpha^{\prime}, \alpha,-\beta\right) \text { of } E \oplus E \oplus H .
$$

5.2. We consider now the cohomology with values in $\mathbb{Z} / 2$. For a lattice $M$, we will write $M_{2}:=M / 2 M$. The scalar product of $M$ induces a product $M_{2} \otimes M_{2} \rightarrow \mathbb{Z} / 2$; if moreover $M$ is even, there is a natural quadratic form $q: M_{2} \rightarrow \mathbb{Z} / 2$ associated with that product, defined by $q(m)=\frac{1}{2} \tilde{m}^{2}$, where $\tilde{m} \in M$ is any lift of $m \in M_{2}$. In particular, $H_{2}$ contains a unique element $\varepsilon$ with $q(\varepsilon)=1$ : it is the class of $e+f$ where $(e, f)$ is a hyperbolic basis of $H$.

Using the previous isomorphism we identify $\mathrm{H}^{2}(X, \mathbb{Z} / 2)$ with $E_{2} \oplus E_{2} \oplus H_{2}$.

Proposition 5.3. The image of $\pi^{*}: \mathrm{H}^{2}(S, \mathbb{Z} / 2) \rightarrow \mathrm{H}^{2}(X, \mathbb{Z} / 2)$ is $\delta\left(E_{2}\right) \oplus(\mathbb{Z} / 2) \varepsilon$.

Proof. This image is invariant under $\sigma^{*}$, hence is contained in $\delta\left(E_{2}\right) \oplus H_{2}$; by Proposition $3.6(i)$ it is 11-dimensional, hence a hyperplane in $\delta\left(E_{2}\right) \oplus H_{2}$, containing $\delta\left(E_{2}\right)$ (which is spanned by the classes coming from $\mathrm{H}^{2}(S, \mathbb{Z})$ ). So $\pi^{*} \mathrm{H}^{2}(S, \mathbb{Z} / 2)$ is spanned by $\delta\left(E_{2}\right)$ and a nonzero element of $H_{2}$; it suffices to prove that this element is $\varepsilon$. Since the elements of $\mathrm{H}^{2}(S, \mathbb{Z} / 2)$ which do not come from $\mathrm{H}^{2}(S, \mathbb{Z})$ have square 1 (3.2), this is a consequence of the following lemma.

Lemma 5.4. For every $\alpha \in \mathrm{H}^{2}(S, \mathbb{Z} / 2), q\left(\pi^{*} \alpha\right)=\alpha^{2}$. 
Proof. This proof has been shown to me by J. Lannes. The key ingredient is the Pontryagin square, a cohomological operation

$$
\mathcal{P}: \mathrm{H}^{2 m}(M, \mathbb{Z} / 2) \longrightarrow \mathrm{H}^{4 m}(M, \mathbb{Z} / 4)
$$

defined for any reasonable topological space $M$ and satisfying a number of interesting properties (see [M-T], ch. 2, exerc. 1). We will state only those we need in the case of interest for us, namely $m=2$ and $M$ is a compact oriented 4-manifold. We identify $\mathrm{H}^{4}(M, \mathbb{Z} / 4)$ with $\mathbb{Z} / 4$; then $\mathcal{P}: \mathrm{H}^{2}(M, \mathbb{Z}) \rightarrow \mathbb{Z} / 4$ satisfies:

a) For $\alpha \in \mathrm{H}^{2}(M, \mathbb{Z} / 2)$, the class of $\mathcal{P}(\alpha)$ in $\mathbb{Z} / 2$ is $\alpha^{2}$;

b) If $\alpha \in \mathrm{H}^{2}(M, \mathbb{Z} / 2)$ comes from $\tilde{\alpha} \in \mathrm{H}^{2}(M, \mathbb{Z})$, then $\mathcal{P}(\alpha)=\tilde{\alpha}^{2}$ (mod. 4$)$. In particular, if $M$ is a K3 surface, we have $\mathcal{P}(\alpha)=2 q(\alpha)$ in $\mathbb{Z} / 4$.

Coming back to our situation, let $\alpha \in \mathrm{H}^{2}(S, \mathbb{Z} / 2)$. We have in $\mathbb{Z} / 4$ :

$$
\begin{aligned}
\mathcal{P}\left(\pi^{*} \alpha\right) & =2 \mathcal{P}(\alpha) & & \text { by functoriality } \\
& =2 \alpha^{2} & & \text { by a }), \text { and } \\
\mathcal{P}\left(\pi^{*} \alpha\right) & =2 q\left(\pi^{*} \alpha\right) & & \text { by b). }
\end{aligned}
$$

Comparing the two last lines gives the lemma.

Corollary 5.5. The kernel of $\pi_{*}: H_{2} \rightarrow\left\{0, k_{S}\right\}$ is $\{0, \varepsilon\}$.

Proof. By Proposition $5.3 \varepsilon$ belongs to $\operatorname{Im} \pi^{*}$, hence $\pi_{*} \varepsilon=0$. It remains to check that $\pi_{*}$ is nonzero on $\mathrm{H}^{1}\left(\mathbb{Z} / 2, \mathrm{H}^{2}(X, \mathbb{Z})\right) \cong H_{2}$. We know that there is an element $\alpha \in \mathrm{H}^{2}(X, \mathbb{Z})$ with $\pi_{*} \alpha=K_{S}$ (Prop. $\left.3.6(i i)\right)$; it belongs to $\operatorname{Ker}\left(1+\sigma^{*}\right)$, hence defines an element $\bar{\alpha}$ of $\mathrm{H}^{1}\left(\mathbb{Z} / 2, \mathrm{H}^{2}(X, \mathbb{Z})\right)$ with $\pi_{*} \bar{\alpha} \neq 0$.

Corollary 5.6. Let $\lambda \in \mathrm{H}^{2}(X, \mathbb{Z})$. The following conditions are equivalent:

(i) $\pi_{*} \lambda=0$ and $\lambda \notin\left(1-\sigma^{*}\right)\left(\mathrm{H}^{2}(X, \mathbb{Z})\right)$;

(ii) $\sigma^{*} \lambda=-\lambda$ and $\lambda^{2} \equiv 2$ (mod. 4$)$.

Proof. Write $\lambda=\left(\alpha, \alpha^{\prime}, \beta\right) \in E \oplus E \oplus H$; let $\bar{\beta}$ be the class of $\beta$ in $H_{2}$. Both conditions imply $\sigma^{*} \lambda=-\lambda$, hence $\alpha^{\prime}=-\alpha$. Since $(\alpha,-\alpha)=\left(1-\sigma^{*}\right)(\alpha, 0)$ and $2 \beta=\left(1-\sigma^{*}\right)(\beta)$, the conditions of $(i)$ are equivalent to $\pi_{*} \bar{\beta}=0$ and $\bar{\beta} \neq 0$, that is, $\bar{\beta}=\varepsilon$ (Corollary 5.5). On the other hand we have $\lambda^{2}=2 \alpha^{2}+\beta^{2} \equiv 2 q(\bar{\beta})$ (mod. 4), hence (ii) is also equivalent to $\bar{\beta}=\varepsilon$.

This allows us to rephrase Corollary 4.3 in a simpler way:

Corollary 5.7. We have $\pi^{*} b_{S}=0$ if and only if there exists a line bundle $L$ on $X$ with $\sigma^{*} L=L^{-1}$ and $c_{1}(L)^{2} \equiv 2(\bmod .4)$.

Remark.- My original proof of (5.3-5) was less direct and less general, but still perhaps of some interest. The key point is to show that on $H_{2} q$ takes the value 1 exactly on the nonzero element of $\operatorname{Ker} \pi_{*}$, or equivalently that an element $\alpha \in \mathrm{H}_{2}$ with $\pi_{*} \alpha=k_{S}$ satisfies $q(\alpha)=0$. Using deformation theory (see (6.1) below), one can assume that $\alpha$ comes from a class in $\operatorname{Pic}(X)$. To conclude I applied the following lemma:

Lemma 5.8. Let $L$ be a line bundle on $X$ with $\operatorname{Nm}(L)=K_{S}$. Then $c_{1}(L)^{2}$ is divisible by 4 . 
Proof. Consider the rank 2 vector bundle $E=\pi_{*}(L)$. The norm induces a nondegenerate quadratic form $N: \mathrm{Sym}^{2} E \rightarrow K_{S}$ ([EGA2], 6.5.5). In particular, $N$ induces an isomorphism $E \stackrel{\sim}{\sim} E^{*} \otimes K_{S}$, and defines a pairing

$$
\mathrm{H}^{1}(S, E) \otimes \mathrm{H}^{1}(S, E) \rightarrow \mathrm{H}^{2}\left(S, K_{S}\right) \cong \mathbb{C}
$$

which is skew-symmetric and non-degenerate. Thus $h^{1}(E)$ is even; since $h^{0}(E)=$ $h^{2}(E)$ by Serre duality, $\chi(E)$ is even, and so is $\chi(L)=\chi(E)$. By Riemann-Roch this implies that $\frac{1}{2} c_{1}(L)^{2}$ is even.

\section{The vanishing of $\pi^{*} b_{\mathrm{S}}$ on the moduli space}

6.1. We briefly recall the theory of the period map for Enriques surfaces, due to Horikawa (see [BHPV], ch. VIII, or [N]). We keep the notations of (5.1). We denote by $L$ the lattice $E \oplus E \oplus H$, and by $L^{-}$the $(-1)$-eigenspace of the involution $\rho$ : $\left(\alpha, \alpha^{\prime}, \beta\right) \mapsto\left(\alpha^{\prime}, \alpha,-\beta\right)$, that is, the submodule of elements $(\alpha,-\alpha, \beta)$.

A marking of the Enriques surface $S$ is an isometry $\varphi: \mathrm{H}^{2}(X, \mathbb{Z}) \rightarrow L$ which conjugates $\sigma^{*}$ to $\rho$. The line $\mathrm{H}^{2,0} \subset \mathrm{H}^{2}(X, \mathbb{C})$ is anti-invariant under $\sigma^{*}$, so its image by $\varphi_{\mathbb{C}}: \mathrm{H}^{2}(X, \mathbb{C}) \rightarrow L_{\mathbb{C}}$ lies in $L_{\mathbb{C}}^{-}$. The corresponding point $[\omega]$ of $\mathbb{P}\left(L_{\mathbb{C}}^{-}\right)$is the period $\wp(S, \varphi)$. It belongs to the domain $\Omega \subset \mathbb{P}\left(L_{\mathbb{C}}^{-}\right)$defined by the equations

$$
(\omega \cdot \omega)=0 \quad(\omega \cdot \bar{\omega})>0 \quad(\omega \cdot \lambda) \neq 0 \quad \text { for all } \lambda \in L^{-} \text {with } \lambda^{2}=-2 .
$$

This is an analytic manifold, which is the moduli space for marked Enriques surfaces. To each class $\lambda \in L^{-}$we associate the hypersurface $H_{\lambda}$ of $\Omega$ defined by $(\lambda \cdot \omega)=0$.

Proposition 6.2. We have $\pi^{*} b_{S}=0$ if and only if $\wp(S, \varphi)$ belongs to one of the hypersurfaces $H_{\lambda}$ for some vector $\lambda \in L^{-}$with $\lambda^{2} \equiv 2$ (mod. 4).

Proof. The period point $\wp(S, \varphi)$ belongs to $H_{\lambda}$ if and only if $\lambda$ belongs to $c_{1}(\operatorname{Pic}(X))$; by Corollary 5.7, this is equivalent to $\pi^{*} b_{S}=0$.

To get a complete picture we want to know which of the $H_{\lambda}$ are really needed:

Lemma 6.3. Let $\lambda$ be a primitive element of $L^{-}$.

(i) The hypersurface $H_{\lambda}$ is non-empty if and only if $\lambda^{2}<-2$.

(ii) If $\mu$ is another primitive element of $L^{-}$with $H_{\mu}=H_{\lambda} \neq \varnothing$, then $\mu= \pm \lambda$.

Proof. Let $W$ be the subset of $L_{\mathbb{C}}^{-}$defined by the conditions $\omega^{2}=0, \omega \cdot \bar{\omega}>0$. If we write $\omega=\alpha+i \beta$ with $\alpha, \beta \in L_{\mathbb{R}}^{-}$, these conditions translate as $\alpha^{2}=\beta^{2}>0, \alpha \cdot \beta=0$. Thus $W \cap \lambda^{\perp} \neq \varnothing$ is equivalent to the existence of a positive 2-plane in $L_{\mathbb{R}}^{-}$orthogonal to $\lambda$. Since $L^{-}$has signature $(2,10)$, this is also equivalent to $\lambda^{2}<0$.

If $W \cap \lambda^{\perp}$ is non-empty, $\lambda^{\perp}$ is the only hyperplane containing it, and $\mathbb{C} \lambda$ is the orthogonal of $\lambda^{\perp}$ in $L^{-}$. Then $\lambda$ and $-\lambda$ are the only primitive vectors of $L^{-}$contained in $\mathbb{C} \lambda$. In particular $\lambda$ is determined up to sign by $H_{\lambda}$, which proves $(i i)$.

Let us prove $(i)$. We have seen that $H_{\lambda}$ is empty for $\lambda^{2} \geq 0$, and also for $\lambda^{2}=-2$ by definition of $\Omega$. Assume $\lambda^{2}<-2$ and $H_{\lambda}=\varnothing$; then $H_{\lambda}$ must be contained in one of the hyperplanes $H_{\mu}$ with $\mu^{2}=-2$; by (ii) this implies $\lambda= \pm \mu$, a contradiction. 
6.4. Let $\Gamma$ be the group of isometries of $L^{-}$. The group $\Gamma$ acts properly discontinuously on $\Omega$, and the quotient $\mathcal{M}=\Omega / \Gamma$ is a quasi-projective variety. The image in $\mathcal{M}$ of the period $\wp(S, \varphi)$ does not depend on the choice of $\varphi$; let us denote it by $\wp(S)$. The map $S \mapsto \wp(S)$ induces a bijection between isomorphism classes of Enriques surfaces and $\mathcal{M}$; the variety $\mathcal{M}$ is a (coarse) moduli space for Enriques surfaces.

Corollary 6.5. The surfaces $S$ for which $\pi^{*} b_{S}=0$ form an infinite, countable union of (non-empty) hypersurfaces in the moduli space $\mathcal{M}$.

Proof. Let $\Lambda$ be the set of primitive elements $\lambda$ in $L^{-}$with $\lambda^{2}<-2$ and $\lambda^{2} \equiv$ 2 (mod. 4). For $\lambda \in \Lambda$, let $\mathcal{H}_{\lambda}$ be the image of $H_{\lambda}$ in $\mathcal{M}$; the argument of [BHPV], ch. VIII, Cor. 20.7 shows that $\mathcal{H}_{\lambda}$ is an algebraic hypersurface in $\mathcal{M}$. By Proposition 6.2 and Lemma 6.3 the surfaces $S$ with $\pi^{*}\left(b_{S}\right)=0$ form the subset $\bigcup_{\lambda \in \Lambda} \mathcal{H}_{\lambda}$. By Lemma 6.3 (ii) we have $\mathcal{H}_{\lambda}=\mathcal{H}_{\mu}$ if and only if $\mu= \pm g \lambda$ for some element $g$ of $\Gamma$. This implies $\lambda^{2}=\mu^{2}$; but $\lambda^{2}$ can be any number of the form $-2 k$ with $k$ odd $>1$ (take for instance $\lambda=e-k f$, where $(e, f)$ is a hyperbolic basis of $H$ ), so there are infinitely many distinct hypersurfaces among the $\mathcal{H}_{\lambda}$.

\section{Acknowledgements}

I am indebted to J.-L. Colliot-Thélène for explaining the problem to me, and for very useful discussions and comments. I am grateful to J. Lannes for providing the topological proof of Lemma 5.4.

\section{References}

[BHPV] W. Barth, K. Hulek, C. Peters, A. Van de Ven: Compact complex surfaces. 2nd edition. Ergebnisse der Mathematik und ihrer Grenzgebiete, 4. Springer-Verlag, Berlin (2004).

[EGA2] A. Grothendieck: Éléments de géométrie algébrique II. Publ. Math. IHES 8 (1961).

[G] A. Grothendieck: Le groupe de Brauer I-II. Dix Exposés sur la Cohomologie des Schémas, pp. 46-87; North-Holland, Amsterdam (1968).

[H-S] D. Harari, A. Skorobogatov: Non-abelian descent and the arithmetic of Enriques surfaces. Intern. Math. Res. Notices 52 (2005), 3203-3228.

[dJ] A.J. de Jong: A result of Gabber. Preprint.

[M-S] J. Milnor, J. Stasheff: Characteristic classes. Annals of Math. Studies 76. Princeton University Press, Princeton (1974).

[M-T] R. Mosher, M. Tangora: Cohomology operations and applications in homotopy theory. Harper \& Row, New York-London (1968).

[N] Y. Namikawa: Periods of Enriques surfaces. Math. Ann. 270 (1985), no. 2, 201-222.

[S] A. Skorobogatov: On the elementary obstruction to the existence of rational points. Math. Notes 81 (2007), no. 1, 97-107.

Laboratoire J.-A. Dieudonné, UMR 6621 du CNRS, Université de Nice, Parc Valrose, F-06108 Nice Cedex 2, France

E-mail address: arnaud.beauville@unice.fr 